\title{
Towards Adaptive Text Summarization: How Does Compression Rate Affect Summary Readability of L2 Texts?
}

\author{
Tatiana Vodolazova, Elena Lloret \\ Dept. of Software and Computing Systems \\ University of Alicante \\ Apdo. de Correos 99 \\ E-03080, Alicante, Spain \\ \{tvodolazova,elloret\}@dlsi.ua.es
}

\begin{abstract}
This paper addresses the problem of readability of automatically generated summaries in the context of second language learning. For this we experimented with a new corpus of level-annotated simplified English texts. The texts were summarized using a total of 7 extractive and abstractive summarization systems with compression rates of $20 \%, 40 \%, 60 \%$ and $80 \%$. We analyzed the generated summaries in terms of lexical, syntactic and length-based features of readability, and concluded that summary complexity depends on the compression rate, summarization technique and the nature of the summarized corpus. Our experiments demonstrate the importance of choosing appropriate summarization techniques that align with users needs and language proficiency.
\end{abstract}

\section{Introduction}

It is often the case that people, at some point in their lives, are incapable of benefiting from available information due to various aspects of text complexity resulting from domain-specific terminology and grammatical structure complexity. The literature has identified specific instances of this, such as: diabetes patients with no medical training who try to make sense of recent scientific advances in the treatment of the disease (Ong et al., 2008); elderly people forced to embrace the technical terminology of the digital age when using computers and mobile phones (Li and Perkins, 2007); parents trying to decipher the latest slang words used by their teenage children (Vizgirdaite, 2009); people with different degrees of learning difficulties such as aphasia (Carroll et al., 1998), dyslexia (Rello et al., 2012) or autism (Štajner et al., 2012); and, second languag (L2) learners trying to infer the meaning of idioms from the literal meaning of their constituents (Charteris-Black, 2002).

Extensive research has been carried out in the field of automatic text simplification and text enrichment (Rello et al., 2014; Aranzabe et al., 2012; Woodsend and Lapata, 2011; Thomas and Anderson, 2012; Barbu et al., 2015). But only a few studies integrate them into other applications of natural language processing (NLP), such as, for example, in text summarization for the purpose of improving readability. Text summarization is not considered to be primarily a simplification task. However, it becomes useful when long documents are involved as it aims to reduce text processing time and thus to access quicker the main concepts of the document. In this context most of the studies apply text simplification to reduce redundant or less important information and to increase the informativeness of extractive summaries, but not their readability (Jing, 2000). Lloret et al. (2019) point out that summaries are rarely evaluated for readability. But text summarization can clearly benefit from readability assessment to better serve its purpose of saving reading time and to avoid the generation of incomprehensible summaries. We take this idea further and suggest that to maintain an optimal level of summary complexity and to adapt it in a personalized way according to user needs and language proficiency, the summarization aproach needs to identify and integrate the necessary degree of simplification.

This paper presents the initial study of ongoing research on the development of an abstractive text summarization approach that can adapt generated summaries to user language proficiency and cognitive abilities. In this study, we rely on the fact that texts for L2 learners are written in a well-structured manner with clear style. Unlike first language texts, these texts, in addition to their semantic readability features, include a broader range of syntactic features, thereby providing an 
overall richer set of readability metrics for examination (Heilman et al., 2007). Once identified these features can be used to measure variation in readability of automatically generated summaries.

For this purpose we harvested a corpus of texts for L2 learners of English that are classified into 7 levels according to language proficiency. This dataset provides an appropriate setup to explore the distribution of different readability characteristics across the levels and to study how these characteristics change when summarization is applied.

The main contributions of this paper are:

- we experiment with a new dataset of graded L2 learner texts that can be used both for text simplification and text summarization tasks;

- we test a number of summarization approaches on this corpus and demonstrate that compression rate always affects the complexity of generated summaries;

- we show that the domain of the corpus affects the results;

- we prove that the complexity of generated summaries varies depending on the summarization technique and the readability metric.

\section{Related work}

The present study spans two fields of NLP: text summarization and readability assessment as a part of the text simplification process.

Only a few studies address the problem of how text summarization affects summary readability. Petersen and Ostendorf (2007) are among the first to point out that text summarization techniques alone do not control the degree of readability of generated summaries because sentences with challenging vocabulary and complex grammatical structures may be chosen. Lloret et al. (2019) further explore this idea, showing that text summarization does not maintain the same degree of text complexity as the original document. However, based on their experiments with a corpus of unsimplified newswire documents for native speakers DUC $2002^{1}$ and contrary to the observation of Petersen and Ostendorf (2007), they conclude that on some readability metrics the summaries generated with the compression rate of $20 \%$ score better and are easier to comprehend than the original texts. For their experiments Lloret et al. (2019) used a

\footnotetext{
${ }^{1}$ http://duc.nist.gov/
}

total of 9 lexical and length-based readability features and a modular extractive text summarization approach that allowed the testing of how anaphora resolution, word sense disambiguation and textual entailment affect the readability of summaries.

Only a handful of text summarization methods so far have integrated readability assessment to select not only the most informative, but also the most comprehensible sentences. Nandhini and Balasundaram (2014) designed one of such approaches. They represent each document as a set of 4 informative features (sentence position, title similarity, etc.) and 5 readability features (word length, sentence length, etc.) and treat summarization as an optimization problem to maximize the average informative score of the summary and to improve its readability. However, their set of readability features is small and they do not study the relative importance of each feature with respect to the corpus or the target language proficiency level.

In recent years, several studies appeared that address readability assessment and text simplification for L2 learners. Vajjala and Lučić (2018) compiled the OneStopEnglish corpus of simplified level-annotated news articles for L2 learners with comparable texts across all levels. Xia et al. (2016) trained a machine learning algorithm for readability assessment on past Cambridge English Exam papers. Their set of readability features includes data from English Vocabulary Profile, an online vocabulary resource with integrated grading scale based on the Common European Framework of Reference for Languages (CEFR) (Council of Europe, 2001).

Our study has been inspired by the aforementioned research and further expands on the idea of Lloret et al. (2019) by experimenting with a graded simplified corpus of texts for L2 learners of English, adding vocabulary-based and syntactic families of readability features, including an abstractive summarization system and testing these metrics with 4 different compression rates.

\section{Data}

The goal of this paper is to provide an initial analysis on how automatic text summarization affects readability for L2 learners with the long term goal of integrating these findings into an abstractive text summarization approach capable of adapting generated summaries to user language proficiency, knowledge and cognitive abilities. This requires a 


\begin{tabular}{|l|c|c|c|c|c|c|c|}
\hline Level & $\mathbf{0}$ & $\mathbf{1}$ & $\mathbf{2}$ & $\mathbf{3}$ & $\mathbf{4}$ & $\mathbf{5}$ & $\mathbf{6}$ \\
\hline \hline \# of docs & 251 & 251 & 251 & 251 & 250 & 250 & 250 \\
CEFR & A2 & A2 & A2 & A2-B1 & B1-B2 & B2 & C1-C2 \\
\% of docs & $64.94 \%$ & $45.02 \%$ & $23.11 \%$ & $61.35 \%$ & $78.40 \%$ & $54.40 \%$ & $40.80 \%$ \\
Difficulty & low & low-med & med & pre-int & pre-int/int & int & upper-int \\
\hline
\end{tabular}

Table 1: Statistics for the BNE corpus

corpus of simplified texts that are adapted to different reading proficiency levels and also include the original source documents. To the best of our knowledge there is no such freely available levelannotated corpus of learner's materials that can also be used for text summarization.

Motivated by this fact we harvested a corpus of texts for English learners from Breaking News English website ${ }^{2}$. Breaking News English (BNE) is a website with different resources for English language teachers created and maintained since November 2004 by an experienced ESL/EFL teacher Sean Banville ${ }^{3}$. This website was nominated for a British Council ELTons award in the category "Innovation in Learner Resources" in 2014. We obtained permission from the author to use it for research purposes. The site contains materials classified into seven levels from 0 to 6 that roughly correspond to CEFR grading scale, comprising levels from A2 to C2 (see Table 3). A new lesson, containing quizzes, reading and listening activities, appears every two days. The main component of each lesson is a piece of news from 120 to 250 words long, depending on the level. Texts cover a broad range of topics, but try to avoid more emotive and sensitive ones (Banville, 2005). As the vocabulary difficulty depends on the topic, each news article topic is assigned to one of two groups, representing linguistic levels 0 to 3 or 4 to 6 , respectively. The grading process is the following: first, the author manually creates a text for level 3 or 6 and then makes the easier levels by reducing sentence length, simplifying grammar, introducing easier vocabulary and avoiding idioms. We will revisit this grading policy in Section 4.2, as it affects readability statistics of the corpus.

The distinguishing features of this resource are:

- each text is available at different levels of complexity.

- each text can be considered as a summary of a set of news articles that can be extracted from the provided URLs.

\footnotetext{
${ }^{2}$ https://breakingnewsenglish.com/

${ }^{3}$ https://www.linkedin.com/in/seanbanville/
}

As mentioned in Section 2 the OneStopEnglish corpus (Vajjala and Lučić, 2018) is a similar resource for readability assessment that contains the same texts rewritten for elementary, intermediate or advanced reading proficiency levels. However, given the 7 levels from the BNE dataset we expect to be able to identify the more subtle readability differences between them. Going forward, with access to the original news articles via URLs, we will be able to develop and test an adaptive multidocument summarization approach on this corpus.

The BNE website has been evolving since its creation in 2004, thereby level annotation is available only starting from July 2013. We harvested all the suitable data, but for this initial research we used a subset from March 2016 to January 2019 , resulting in 1,754 news articles in total and 250/251 articles for each of the 7 levels. Table 1 shows further statistics of the corpus where CEFR and Difficulty rows reflect grading information provided on the resource's website.

To contrast BNE's website CEFR level annotation with the CEFR annotation of other state-ofthe-art resources, we evaluated our corpus with the readability assessment method developed by Xia et al. (2016) and trained on past Cambridge English Exam papers and on the set of 100 additionally annotated news articles. The row $\%$ of docs illustrates how many texts were identified by this method as belonging to the indicated CEFR level.

\section{Readability}

\subsection{Features}

The most recent research on readability assessment uses machine learning based approaches in combination with a broad set of linguistic features. Such sets of features are usually organized into families that share similar linguistic properties. They typically include length-based, syntactic, lexico-semantic and discourse-based features, among others. Experiments show that different families, or even individual features, affect the accuracy of the classifier in a different manner (Xia et al., 2016). 
Following previous research on assessing readability of summaries by Lloret et al. (2019), we selected the same set of 9 readability features. However, after detailed analysis of the set and the revision of other research in readability assessment of L2 texts, we added further lexical and syntactic features (Xia et al., 2016; Heilman et al., 2007). The rationale for this was twofold. First, experiments show that these families of features significantly improve performance of classifiers and therefore help to correctly identify the grade of text complexity (Pitler and Nenkova, 2008). Second, the initial set of readability metrics would not be capable of grasping grammatical and vocabulary changes that are an integral part of second language acquisition. To cover these aspects of text complexity we added 12 more features ${ }^{4}$ including the revised Dale-Chall formula (Chall and Dale, 1995). This formula calculates the proportion of words that do not belong to the list of 3,000 familiar words. Some studies view this formula as a simplified version of a language model (CollinsThompson and Callan, 2004). The complete set of 21 features, by families, is described below.

Traditional Features This family includes superficial length-based features and traditional readability formulas that are easy to compute, but provide a competitive baseline.

- Flesch Reading Ease (FRE) (Flesch, 1948)

- Avg. Word Length (AWL)

- Mean Length of a Sentence (MLS)

- Avg. Number of Sentences (ANS)

- Avg. Text Length in Tokens (ANT)

Lexical Features Our final feature set does not include discourse features, although 4 already integrated features from the original feature set of Lloret et al. (2019) based on noun and proper noun ratios may be related to the entity-density features, which could cast some light on the discourse properties of the corpus (Feng et al., 2009).

- Word variation index (OVIX) is a variety of type-token ratio measure (Hultman and Westman, 1977)

- Revised Dale-Chall formula (DC)

- Proper Noun Ratio (PNR)

- Avg. Number of Unique Proper Nouns (uPNR)

\section{- Noun Ratio (NR)}

- Pronoun Ratio (PR)

\footnotetext{
${ }^{4}$ We indicate them with an $*$ in Table 2
}

Syntactic Features Heilman et al. (2007) emphasize that grammatical features may play a more important role in readability assessment for the L2 learners than for the native speakers. Following their example, we calculate the last 4 features in this family on a per word basis.

- Parse Tree Depth (PTD)

- Noun Phrase Ratio (NPR)

- Verb Phrase Ratio (VPR)

- Adjective Phrase Ratio (ADJPR)

- Adverbial Phrase Ratio (ADVPR)

- Avg. number of SBARs per sentence (SBAR)

- Ratio of Passive Voice constructions (PV)

- Avg. number of Relative Clauses (RC)

- Past Participles (VBN)

- Modal verbs (MD)

\subsection{BNE Readability Statistics}

In Section 3 we explained how Sean Banville manually creates the news articles for each complexity level. His grading scheme does not correspond one-to-one to other established classifications, such as for example CEFR level annotation. To analyze how readability varies across the $7 \mathrm{lev}$ els, we extracted statistics from the BNE corpus for each of the 21 features. Table 2 contains average values by level for each of the selected readability measures and Table 3 Pearson correlation coefficient.

Length-based readability features reveal irregularities in the size of articles between levels 3 and 4 when the more complex topic replaces the easier one. One can observe that the average number of tokens and sentences per article, as well as the mean sentence length (ANS, ANT, MLS) gradually increase from level 0 to level 3, but then in level 4 decrease almost to the values of level 1 , again increasing and surpassing previous values up until level 6. It affects FRE formula and introduces the same irregularity in its values. These size differences are intended by the author. Indeed, he states on his website that levels 4 and 5 texts are shorter than levels 2 and 3 respectively. Average word length is the only length-based feature that grows linearly and slightly smooths out the values of FRE.

This tendency in length-based features also affects syntactic features, since more complex syntactic constructions tend to contain more words. Considering these findings, none of the discussed features in isolation, except for AWL, could be 


\begin{tabular}{|l|c|c|c|c|c|c|c|}
\hline Level & $\mathbf{0}$ & $\mathbf{1}$ & $\mathbf{2}$ & $\mathbf{3}$ & $\mathbf{4}$ & $\mathbf{5}$ & $\mathbf{6}$ \\
\hline \hline FRE & $\mathbf{6 9 . 1 2 3}$ & $\mathbf{6 4 . 0 6 0}$ & $\mathbf{5 9 . 2 3 5}$ & $\mathbf{5 3 . 9 5 1}$ & $\mathbf{5 5 . 4 7 0}$ & $\mathbf{5 0 . 0 7 3}$ & $\mathbf{4 2 . 0 1 1}$ \\
AWL & 4.621 & 4.681 & 4.754 & 4.839 & 4.860 & 4.937 & 5.063 \\
MLS & $\mathbf{9 . 9 9 5}$ & $\mathbf{1 2 . 1 8 6}$ & $\mathbf{1 4 . 2 9 2}$ & $\mathbf{1 6 . 8 4 6}$ & $\mathbf{1 4 . 0 2 7}$ & $\mathbf{1 6 . 6 2 3}$ & $\mathbf{2 0 . 4 3 2}$ \\
ANS* & 10.912 & 12.498 & 13.677 & 14.040 & 10.716 & 11.476 & 11.160 \\
ANT & $\mathbf{1 0 6 . 0 0 0}$ & $\mathbf{1 4 7 . 7 0 1}$ & $\mathbf{1 8 8 . 8 2 9}$ & $\mathbf{2 2 9 . 0 1 6}$ & $\mathbf{1 4 4 . 5 7 2}$ & $\mathbf{1 8 3 . 5 8 8}$ & $\mathbf{2 2 0 . 1 6 0}$ \\
\hline OVIX & 45.091 & 45.855 & 46.258 & 47.009 & 50.507 & 51.076 & 52.702 \\
DC* & $\mathbf{7 . 1 0 6}$ & $\mathbf{7 . 4 3 8}$ & $\mathbf{7 . 7 2 0}$ & $\mathbf{8 . 0 0 0}$ & $\mathbf{8 . 2 1 6}$ & $\mathbf{8 . 5 4 0}$ & $\mathbf{8 . 9 8 9}$ \\
PNR & 0.053 & 0.052 & 0.052 & 0.055 & 0.048 & 0.049 & 0.052 \\
uPNR & 0.033 & 0.033 & 0.033 & 0.036 & 0.033 & 0.034 & 0.038 \\
NR & 0.311 & 0.304 & 0.301 & 0.299 & 0.305 & 0.302 & 0.300 \\
PR & 0.072 & 0.069 & 0.067 & 0.063 & 0.066 & 0.062 & 0.058 \\
\hline PTD* & $\mathbf{8 . 5 4 2}$ & $\mathbf{9 . 3 2 0}$ & $\mathbf{9 . 9 9 7}$ & $\mathbf{1 0 . 8 2 1}$ & $\mathbf{9 . 7 6 6}$ & $\mathbf{1 0 . 4 3 4}$ & $\mathbf{1 1 . 4 6 9}$ \\
NPR* & 2.650 & 2.893 & 3.060 & 3.258 & 2.966 & 3.141 & 3.421 \\
VPR* & 1.067 & 1.091 & 1.114 & 1.130 & 1.106 & 1.136 & 1.160 \\
ADJPR* & 0.223 & 0.250 & 0.262 & 0.284 & 0.242 & 0.247 & 0.288 \\
ADVPR & 0.200 & 0.241 & 0.285 & 0.354 & 0.281 & 0.323 & 0.401 \\
SBAR* & 0.337 & 0.401 & 0.460 & 0.527 & 0.420 & 0.476 & 0.542 \\
PV* & 0.005 & 0.006 & 0.007 & 0.008 & 0.009 & 0.009 & 0.011 \\
RC* & 0.006 & 0.008 & 0.009 & 0.010 & 0.008 & 0.009 & 0.010 \\
VBN* & $\mathbf{0 . 1 1 7}$ & $\mathbf{0 . 1 7 1}$ & $\mathbf{0 . 2 2 6}$ & $\mathbf{0 . 3 1 2}$ & $\mathbf{0 . 2 8 5}$ & $\mathbf{0 . 3 5 4}$ & $\mathbf{0 . 4 9 1}$ \\
MD* & 0.257 & 0.256 & 0.261 & 0.265 & 0.240 & 0.235 & 0.233 \\
\hline
\end{tabular}

Table 2: Readability statistics for the BNE corpus

used to correctly identify the level of a BNE document. Among lexical features, we want to point out the OVIX and the DC metrics that together with the AWL suggest that the best approach to automatically assess complexity of this corpus may involve a statistical language model.

Based on the values of Pearson correlation coefficient we reduced our readability set to 3 features including FRE, DC and PTD - one feature per family - as the most reliable readability indicators for the given corpus (see Table 3 ). Since average sentence length is one of the components of FRE, we included FRE and not MSL in this set, even though it has a higher correlation coefficient. We will use this reduced set in the next experiments.

\section{Summarization Methods}

For this research we considered 7 state-of-theart methods with different summarization techniques that include graph-based and frequencybased methods, methods that implement language models, incorporate such heuristics as word sense disambiguation and anaphora resolution and involve abstractive text summarization. Implementations of 2 of them were obtained from the authors (ExL19, AbL15), while the remaining 5 were provided by the sumy framework ${ }^{5}$. Each

\begin{tabular}{|l|c|c|}
\hline \multirow{2}{*}{ Feature } & \multicolumn{2}{|c|}{ Pearson correlation } \\
\cline { 2 - 3 } & $\mathbf{r}$ & p-value \\
\hline \hline FRE & $\mathbf{- 0 . 6 1 9 7}$ & $\mathbf{0}$ \\
AWL & 0.4133 & 0 \\
MLS & $\mathbf{0 . 6 4 5 9}$ & $\mathbf{0}$ \\
ANS & -0.1167 & 0 \\
ANT & 0.6154 & 0 \\
\hline OVIX & 0.4080 & 0 \\
DC & $\mathbf{0 . 5 4 2 9}$ & $\mathbf{0}$ \\
PNR & -0.0197 & -0.410 \\
uPNR & 0.0527 & -0.0272 \\
NR & -0.0597 & -0.0125 \\
PR & -0.1393 & 0 \\
\hline PTD & $\mathbf{0 . 5 4 5 4}$ & $\mathbf{0}$ \\
NPR & 0.4114 & 0 \\
VPR & 0.2279 & 0 \\
ADJPR & 0.0753 & -0.0016 \\
ADVPR & 0.3218 & 0 \\
SBAR & 0.2555 & 0 \\
PV & 0.2430 & 0 \\
RC & 0.1252 & 0 \\
VBN & 0.5199 & 0 \\
MD & -0.0493 & -0.0388 \\
\hline
\end{tabular}

Table 3: Correlation coefficient for the BNE corpus.

\footnotetext{
${ }^{5}$ https://github.com/miso-belica/sumy
} 
method is described in more detail below.

Luhn's classical technique was one of the first summarization algorithms ranking sentences on word and phrase frequencies (Luhn, 1958). It weighs each sentence according to the number of significant words it contains ignoring high frequency common words from a stop word list.

SumBasic (Nenkova and Vanderwende, 2005) is another frequency-based summarizer that incorporates context information. It assumes that distribution of words in a human summary is similar to that of the original text. The authors reported that it outperformed many of the DUC 2004 systems, so it is frequently used in the literature as a baseline summarizer.

KLSum (Haghighi and Vanderwende, 2009) uses Kullback-Leibler divergence to measure the similarity between a sentence and the language model of the document and selects a set of sentences such that the distribution of words in the selected sentences is as similar as possible to the overall distribution of words in the document.

ExL19 $^{6}$ Lloret et al. (2019) designed a modular extractive text summarization approach based on frequencies. For this experiment we selected the combination that includes anaphora resolution, word sense disambiguation and textual entailment, scoring sentences on concept frequencies.

LexRank (Erkan and Radev, 2004) is a graphbased approach that uses cosine similarity of TFIDF vectors to calculate pairwise similarity between two sentences. The final score of each sentence is the sum of the weights of all the edges connected to it. The sentences are ranked by applying PageRank to the resulting graph.

TextRank (Mihalcea and Tarau, 2004) is another graph-based approach that uses PageRank to rank the sentences. In the case of TextRank, the similarity between two sentences is calculated as the number of words they have in common normalized by sentence length. In contrast to LexRank, TextRank recursively changes the weights of the sentences incorporating in this manner how all the sentences in the graph relate to each other.

AbL15 (Lloret et al., 2015) is an abstractive text summarization approach that incorporates the stages of text interpretation, transformation and summary generation. Each text passes through the

\footnotetext{
${ }^{6} \mathrm{We}$ will refer to the systems developed by Lloret et al. using ExL19 for extractive and AbL15 for the abstractive one.
}

process of syntactic simplification that splits complex sentences into shorter ones. Subsequently, the system extracts subject-verb-object triplets, identifies named entities and head nouns in nouns phrases, and supplies all this information to the summarizer. The summarizer scores each sentence representation based on the extracted information. In the final step the system translates each sentence into its surface representation and selects the highest rated sentences with respect to the maximum allowed summary size.

\section{Results}

The final setup of our experiment includes: 7 summarization methods (Luhn, SumBasic, ExL19, LexRank, TextRank, KLSum, AbL15); 4 compression rates $(20 \%, 40 \%, 60 \% \& 80 \%)$; and, 3 readability metrics (FRE, DC and PTD). To evaluate how much readability of generated summaries (sread) differs from that of the original documents (oread) we calculated for each document $i$ the percent deviation $(P D)$

$$
P D_{i}=\frac{\left(\text { sread }_{i}-\text { oread }_{i}\right)}{\text { oread }_{i}} * 100
$$

and then averaged it across the entire set. This value can be both negative and positive and indicates whether the summary is more or less complex than the original text.

Another way to assess the summarizer's performance on readability is to calculate the average absolute deviation from the original text's complexity, in other words from 0 . With this information we can determine the degree to which the summaries, independently from being more complex or simple, differ from the original documents across all the compression rates. Although the complexity of a system may vary across all the compression rates, their average variation may be very small.

$$
A A D_{s}=\frac{\sum_{n=1}^{4}\left|P D_{n}-0\right|}{4}
$$

where $A A D_{s}$ is the average absolute deviation of a given system $s$ and $P D_{n}$ is its percent deviation for the compression rate $n$.

\subsection{Length-based readability results}

Table 4 shows how selected text summarization methods affect length-based readability features. FRE measure depends on the number of tokens per 


\begin{tabular}{|c|c|c|c|c|c|c|c|}
\hline \%compression & Luhn & SumBasic & KLSum & ExL19 & LexRank & TextRank & AbL15 \\
\hline \hline 20 & $-12.36 \%$ & $12.68 \%$ & $-8.80 \%$ & $-4.82 \%$ & $-1.67 \%$ & $-7.20 \%$ & $-12.36 \%$ \\
40 & $-5.24 \%$ & $9.16 \%$ & $4.68 \%$ & $-3.74 \%$ & $2.25 \%$ & $-2.51 \%$ & $-3.38 \%$ \\
60 & $-1.53 \%$ & $6.94 \%$ & $8.04 \%$ & $-1.41 \%$ & $3.45 \%$ & $-0.59 \%$ & $4.32 \%$ \\
80 & $0.95 \%$ & $4.85 \%$ & $7.63 \%$ & $0.19 \%$ & $3.81 \%$ & $1.35 \%$ & $10.16 \%$ \\
\hline Avg. abs. dev. & $5.02 \%$ & $8.41 \%$ & $7.29 \%$ & $2.54 \%$ & $2.80 \%$ & $2.91 \%$ & $7.56 \%$ \\
\hline
\end{tabular}

Table 4: Flesch Reading Ease statistics

\begin{tabular}{|c|c|c|c|c|c|c|c|}
\hline \%compression & Luhn & SumBasic & KLSum & ExL19 & LexRank & TextRank & AbL15 \\
\hline \hline 20 & $6.98 \%$ & $-5.25 \%$ & $2.06 \%$ & $9.67 \%$ & $2.01 \%$ & $3.30 \%$ & $10.65 \%$ \\
40 & $4.69 \%$ & $-3.29 \%$ & $-3.60 \%$ & $7.72 \%$ & $0.68 \%$ & $2.34 \%$ & $6.62 \%$ \\
60 & $2.73 \%$ & $-2.58 \%$ & $-4.37 \%$ & $6.03 \%$ & $-0.52 \%$ & $1.60 \%$ & $2.91 \%$ \\
80 & $0.76 \%$ & $-1.62 \%$ & $-3.59 \%$ & $4.28 \%$ & $-0.79 \%$ & $0.48 \%$ & $0.05 \%$ \\
\hline Avg. abs. dev. & $3.79 \%$ & $3.18 \%$ & $3.41 \%$ & $6.93 \%$ & $1.00 \%$ & $1.93 \%$ & $5.06 \%$ \\
\hline
\end{tabular}

Table 5: Dale-Chall statistics

sentence and syllables per words; the higher the score, the easier is the text. Thus when the percent deviation is negative, the summary is less comprehensible than the original text. This occurs across almost all of the settings where the summary comprises only $20 \%$ of the original text. The only exception is SumBasic that, with higher compression rates $^{7}$, tends to select shorter words and shorter sentences. For this readability feature SumBasic simplifies summaries across all the compression rates. However, other frequency-based summarization systems show a different tendency: Luhn and ExL19 tend to generate more complex summaries with longer words and sentences for almost all the compression rates. Graph-based approach TextRank shows the same tendency. In turn, KLSum and AbL15 for higher compression rates generate more complex summaries and for the lower compression rates more simple ones. The degree of difficulty for compression rate $20 \%$ is almost the same as the degree of simplification for $80 \%$ rate. For example, consider AbL15 that for 20\% rate generated $12.36 \%$ more complex summaries, while for $80 \%$ rate $10.16 \%$ more simple.

The overall average degree of deviation from the readability of the original document also needs to be taken into account. ExL19 has the lowest average absolute deviation. It generates summaries with closest complexity to the original $(2.54 \%)$ across all the compression rates.

Contrary to the findings of Lloret et al. (2019), who showed that for the DUC 2002 corpus, ExL19 generated more comprehensible summaries with

\footnotetext{
${ }^{7}$ Under "higher compression rates" we understand shorter summaries; in our study compression rate of $20 \%$ is the highest
}

respect to FRE, for the BNE corpus, ExL19 tends to select longer words and sentences. We believe the variation in original document complexity is what causes this difference because the BNE coprus contains texts simplified for L2 learners ranging mostly from A2 to B2 CEFR levels, whereas DUC 2002 is comprised of unsimplified newswire documents that are instrinsically more complex. This may indicate that present analysis of selected summarization systems and their impact on readability cannot be extended to other domains beyond L2 learner materials, since the performance of any summarizer depends on the corpus.

\subsection{Lexical readability results}

Lexical complexity of summaries was evaluated with the help of Dale-Chall formula; lower values of this metric indicate easier to comprehend summaries. Results in Table 5 show that in this evaluation SumBasic again simplifies summaries across all the compression rates. KLSum reveals similar values, except for the setting with $20 \%$ compression rate. Luhn, ExL19 and AbL15 include sentences with high percentage of complex words in summaries. In terms of lexical complexity, summarizers based on graphs (LexRank and TextRank) demonstrate the lowest average absolute deviation across all the compression rates and thus maintain lexical complexity of summaries that are closest to the original documents. For the lengthbased readability metric, graph-based approaches were also among the most similar.

\subsection{Syntactic readability results}

Parse tree depth statistics can be found in Table 6. For this feature as well, higher values indicate 


\begin{tabular}{|c|c|c|c|c|c|c|c|}
\hline \hline \%compression & Luhn & SumBasic & KLSum & ExL19 & LexRank & TextRank & AbL15 \\
\hline 20 & $3.13 \%$ & $-6.38 \%$ & $11.37 \%$ & $-13.86 \%$ & $3.47 \%$ & $7.87 \%$ & $-15.21 \%$ \\
40 & $3.03 \%$ & $-3.70 \%$ & $0.05 \%$ & $-8.06 \%$ & $0.71 \%$ & $5.50 \%$ & $-16.63 \%$ \\
60 & $2.33 \%$ & $-2.48 \%$ & $-2.01 \%$ & $-4.78 \%$ & $-0.20 \%$ & $4.02 \%$ & $-19.87 \%$ \\
80 & $1.13 \%$ & $-1.02 \%$ & $-1.73 \%$ & $-2.53 \%$ & $-0.17 \%$ & $2.46 \%$ & $-23.10 \%$ \\
\hline Avg. abs. dev. & $2.41 \%$ & $3.40 \%$ & $3.79 \%$ & $7.31 \%$ & $1.13 \%$ & $4.96 \%$ & $18.70 \%$ \\
\hline
\end{tabular}

Table 6: Parse tree depth statistics.

summaries with more complex syntactic constructions and negative percent of deviation indicates that the respective system selects syntactically less complex sentences. For this feature SumBasic again simplifies summaries with respect to the original documents; and graph-based LexRank, by maintaining the lowest average absolute deviation, preserves the original text complexity. For parse tree depth and the other two readability features, KLSum tends to simplify summaries on lower compression rates, but for the compression rate of $20 \%$ it generates more complex summaries. Due to the integrated syntactic simplification step, abstractive system AbL15 generates sentences with shorter parse trees across all the settings. It also displays the highest average absolute deviation to the original values. Extractive system ExL19 reveals a similar tendency, namely, while selecting more complex sentences in terms of lexical complexity, it tends to include syntactically more simple sentences in summaries.

\section{Conclusion and Future Work}

In this paper we experimented with a new dataset of level-annotated L2 learner texts that can be used both for text simplification and text summarization tasks. We analyzed its syntactic, lexical and length-based readability features and evaluated its level annotation with a machine learning system trained on data annotated by Cambridge exam annotators.

We further conducted a novel analysis on how different extractive and abstractive summarization techniques at different compression rates affect readability of simplified L2 learner texts. Our experiments showed how this impact varied depending on the system used: 1) frequency-based system SumBasic consistently simplified summaries with respect to the original texts across all the compression rates, and thus may be considered a competitive baseline, not only in terms of recall but also readability; 2) graph-based approaches, especially LexRank, tended to maintain the same complexity as the original document; 3) Luhn's classical frequency-based method generated more complex summaries; 4) KLSum method based on Kullback-Leibler divergence produced complex summaries at higher compression rates, while simplifying them at lower compression rates; 5) integration of anaphora resolution, textual entailment and word-sense disambiguation led to syntactically more simple, but lexically more complex summaries; 6) abstractive summarizer AbL15 oversimplified syntactic structures and maintained at the same time a high complexity of lexical readability component. Hence no common pattern among the summarization approaches was identified with respect to the effect of compression rate on readability.

This work has provided an insight on the behaviour of different summarization approaches and permitted the discovery of a necessary dataset, as well as the analysis of the dataset's readability. These findings can be viewed as the first important step for designing a summarization system aimed at people with different levels of language proficiency. Future lines of research will consider integrating second language acquisition and discourse readability metrics.

\section{Acknowledgments}

This research work has been partially funded by the University of Alicante (Spain), Generalitat Valenciana and the Spanish Government through the projects SIIA (PROMETEU/2018/089), LIVING-LANG (RTI2018-094653-B-C22), INTEGER (RTI2018-094649-B-I00) and Red iGLN (TIN2017-90773-REDT). We would also like to thank Ekaterina Kochmar from the University of Cambridge for her help with the readability assessment of the BNE corpus, as well as Sean Banville for the permission to use his website materials and the valuable insights on the process of their creation. 


\section{References}

María Aranzabe, Arantza Ilarraza, and Itziar GonzalezDios. 2012. First Approach to Automatic Text Simplification in Basque. In Proceedings of the Natural Language Processing for Improving Textual Accessibility (NLP4ITA) workshop (LREC 2012). pages $1-8$.

Sean Banville. 2005. Creating ESL/EFL lessons based on news and current events. The Internet TESL Journal 11(9). http://iteslj.org/Techniques/BanvilleNews/.

Eduard Barbu, M. Teresa Martín-Valdivia, Eugenio Martínez-Cámara, and L. Alfonso Ureña López. 2015. Language technologies applied to document simplification for helping autistic people. Expert Syst. Appl. 42(12):5076-5086.

John Carroll, Guido Minnen, Yvonne Canning, Siobhan Devlin, and John Tait. 1998. Practical simplification of english newspaper text to assist aphasic readers. In In Proc. of AAAI-98 Workshop on Integrating Artificial Intelligence and Assistive Technology. pages 7-10.

Jeanne Sternlicht Chall and Edgar Dale. 1995. Readability revisited: The new Dale-Chall readability formula. Brookline Books.

Jonathan Charteris-Black. 2002. Second language figurative proficiency: A comparative study of Malay and English. Applied linguistics 23(1):104-133.

Kevyn Collins-Thompson and James P. Callan. 2004. A Language Modeling Approach to Predicting Reading Difficulty. In HLT-NAACL 2004: Main Proceedings. Association for Computational Linguistics, Boston, Massachusetts, USA, pages 193200. https://www.aclweb.org/anthology/N04-1025.

Council of Europe. 2001. The Common European Framework of Reference for Languages. Cambridge University Press. https://rm.coe.int/1680459f97.

Günes Erkan and Dragomir R. Radev. 2004. Lexrank: Graph-based lexical centrality as salience in text summarization. J. Artif. Int. Res. 22(1):457-479.

Lijun Feng, Noémie Elhadad, and Matt Huenerfauth. 2009. Cognitively motivated features for readability assessment. In Proceedings of the 12th Conference of the European Chapter of the Association for Computational Linguistics. Association for Computational Linguistics, Stroudsburg, PA, USA, EACL '09, pages 229-237.

Rudolph Flesch. 1948. A new readability yardstick. Journal of applied psychology 32(3):221. https://doi.org/10.1037/h0057532.

Aria Haghighi and Lucy Vanderwende. 2009. Exploring content models for multi-document summarization. In Proceedings of Human Language Technologies: The 2009 Annual Conference of the North
American Chapter of the Association for Computational Linguistics. Association for Computational Linguistics, Stroudsburg, PA, USA, NAACL '09, pages 362-370.

Michael Heilman, Kevyn Collins-Thompson, Jamie Callan, and Maxine Eskenazi. 2007. Combining lexical and grammatical features to improve readability measures for first and second language texts. In Human Language Technologies 2007: The Conference of the North American Chapter of the Association for Computational Linguistics; Proceedings of the Main Conference. Association for Computational Linguistics, Rochester, New York, pages 460467. https://www.aclweb.org/anthology/N07-1058.

Tor G. Hultman and Margareta Westman. 1977. Gymnasistsvenska. Liber Lromedel.

Hongyan Jing. 2000. Sentence reduction for automatic text summarization. In Proceedings of the Sixth Conference on Applied Natural Language Processing. Association for Computational Linguistics, Stroudsburg, PA, USA, ANLC '00, pages 310-315. https://doi.org/10.3115/974147.974190.

Yushi Boni Li and Amanda Perkins. 2007. The impact of technological developments on the daily life of the elderly. Technology in society 29(3):361-368.

Elena Lloret, Ester Boldrini, Tatiana Vodolazova, Patricio Martínez-Barco, Rafael Muñoz, and Manuel Palomar. 2015. A novel concept-level approach for ultra-concise opinion summarization. Expert Syst. Appl. 42(20):7148-7156. https://doi.org/10.1016/j.eswa.2015.05.026.

Elena Lloret, Tatiana Vodolazova, Paloma Moreda, Rafael Muñoz, and Manuel Palomar. 2019. Are better summaries also easier to understand? Analyzing text complexity in automatic summarization. In Marina Litvak and Natalia Vanetik, editors, Multilingual text analysis: Challenges, Models, and Approaches, World Scientific, New Jersey, pages 337369.

Hans Peter Luhn. 1958. The automatic creation of literature abstracts. IBM J. Res. Dev. 2(2):159-165. https://doi.org/10.1147/rd.22.0159.

Rada Mihalcea and Paul Tarau. 2004. TextRank: Bringing order into text. In Proceedings of EMNLP 2004. Association for Computational Linguistics, Barcelona, Spain, pages 404411. https://www.aclweb.org/anthology/W04-3252.

Kumaresh Nandhini and Sadhu Ramakrishnan Balasundaram. 2014. Extracting easy to understand summary using differential evolution algorithm. Swarm and Evolutionary Computation 16:19 - 27. https://doi.org/10.1016/j.swevo.2013.12.004.

Ani Nenkova and Lucy Vanderwende. 2005. The impact of frequency on summarization. Microsoft Research, Redmond, Washington, Tech. Rep. MSR-TR2005101. 
Ethel Ong, Jerwin Damay, Gerard Lojico, Kimberly Lu, and Dex Tarantan. 2008. Simplifying text in medical literature. Journal of Research in Science, Computing and Engineering 4(1):37-48.

Sarah E. Petersen and Mari Ostendorf. 2007. Text simplification for language learners: a corpus analysis. In Workshop on Speech and Language Technology in Education.

Emily Pitler and Ani Nenkova. 2008. Revisiting readability: A unified framework for predicting text quality. In Conference on Empirical Methods in Natural Language Processing, EMNLP 2008. ACL, pages 186-195. http://www.aclweb.org/anthology/D08-1020.

Luz Rello, Clara Bayarri, Azuki Gòrriz, Ricardo Baeza-Yates, Saurabh Gupta, Gaurang Kanvinde, Horacio Saggion, Stefan Bott, Roberto Carlini, and Vasile Topac. 2012. Dyswebxia 2.0!: more accessible text for people with dyslexia. Proceedings of the 10th International Cross-Disciplinary Conference on Web Accessibility pages 41-44. https://doi.org/10.1145/2140446.2140455.

Luz Rello, Horacio Saggion, and Ricardo Baeza-Yates. 2014. Keyword highlighting improves comprehension for people with dyslexia. In Proceedings of the 3rd Workshop on Predicting and Improving Text Readability for Target Reader Populations (PITR). Association for Computational Linguistics, Gothenburg, Sweden, pages 30-37. https://www.aclweb.org/anthology/W14-1204.

S. Rebecca Thomas and Sven Anderson. 2012. Wordnet-based lexical simplification of a document. In Jeremy Jancsary, editor, Proceedings of $\mathrm{KON}$ VENS 2012. ÖGAI, pages 80-88.

Sowmya Vajjala and Ivana Lučić. 2018. OneStopEnglish corpus: A new corpus for automatic readability assessment and text simplification. In Proceedings of the Thirteenth Workshop on Innovative Use of NLP for Building Educational Applications. Association for Computational Linguistics, New Orleans, Louisiana, pages 297-304. https://doi.org/10.18653/v1/W18-0535.

Jurgita Vizgirdaite. 2009. Filling the child-parent relationship gap via the parent self-education and intergenerational education on internet slang. Social Sciences (1392-0758) 64(2).

Sanja Štajner, Richard Evans, Constantin Orasan, and Ruslan Mitkov. 2012. What can readability measures really tell us about text complexity? In Luz Rello and Horacio Saggion, editors, Proceedings of the LREC'12 Workshop: Natural Language Processing for Improving Textual Accessibility (NLP4ITA). European Language Resources Association (ELRA), Istanbul, Turkey.

Kristian Woodsend and Mirella Lapata. 2011. Learning to simplify sentences with quasi-synchronous grammar and integer programming. In Proceedings of the 2011 Conference on Empirical Methods in Natural Language Processing. Association for Computational Linguistics, Edinburgh, Scotland, UK., pages 409-420. https://www.aclweb.org/anthology/D11-1038.

Menglin Xia, Ekaterina Kochmar, and Ted Briscoe. 2016. Text readability assessment for second language learners. In Proceedings of the 11th Workshop on Innovative Use of NLP for Building Educational Applications. Association for Computational Linguistics, San Diego, CA, pages 12-22. https://doi.org/10.18653/v1/W16-0502. 